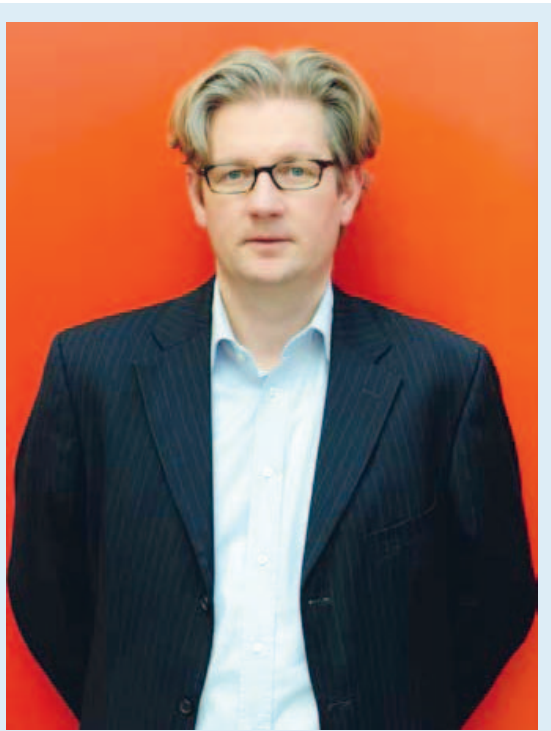

Endlich etabliert

Nicht bei der Behandlung der Patienten entscheidet sich, ob eine neue Richtung der Medizin erfolgreich wird. Weil wir in ökonomischen Zeiten leben, ist es der Souverän, der für oder gegen eine Bezahlung der Kosten in der Grundversicherung ist. Die Ganzheitsmedizin hat diese wichtige Hürde geschafft. Zum 25-jährigen Jubiläum der SCHWEIZERISCHE ZeITSCHRIFT FÜR GANZHEITSMEDIZIN wollten wir von Walter Stüdeli wissen, wie die Zukunftsaussichten der Komplementärmedizin in der Schweiz sind. Er ist einer der besten Kenner der Gesundheitspolitik im Bundeshaus und deshalb der richtige Gesprächspartner, um den Blick nach vorne zu wagen.

\title{
Expertengespräch
}

Schweiz Z Ganzheitsmed 2013;25:12-15

DOI: 10.1159/000346395

\section{Gute Aussichten für die Ganzheitsmedizin}

Die Entscheidung des Souveräns für die Komplementärmedizin hat ihr endgültig zum Durchbruch verholfen. Sie wird zur etablierten Ergänzung der Schulmedizin - die Politik in Bern setzt darauf. Der Lobbyist für Komplementärmedizin Walter Stüdeli sieht die Weichen richtig gestellt.

Was bringt denn 2013 der Ganzheitsmedizin in der Schweiz?

Walter Stüdeli: Im neuen Jahr tut sich eine ganze Menge in der Berner Politik, das Auswirkungen auf die Ganzheits- und Komplementärmedizin in der Schweiz hat. Bundesrat Alain Berset, in dessen Zuständigkeit sie fällt, steht ihr sehr aufgeschlossen gegenüber. Das Jahr 2013 bringt einige Gesetzesentwürfe auf Bundesebene. Wahrscheinlich lanciert der Dachverband Komplementärmedizin auch eine kantonale Initiative in BaselStadt und Basel-Landschaft: Sie will einen Lehrstuhl an der Universität Basel schaffen, der sich mit Lehre und Forschung in Komplementärmedizin beschäftigen soll.

Das Heilmittelgesetz kommt in diesem Jahr ins Parlament. Zusätzlich ist noch das Medizinalberufegesetz in Vorbereitung. Dazu kommt die Anerkennung nationaler Diplome für die nichtärztlichen Therapeuten. Diese können dann beim Bundesamt für Berufsbildung und Technologie genehmigt werden. Es ist also einiges am Laufen und auch einige Weichen werden 2013 gestellt.

\section{Der Zug fährt in eine positive Richtung?}

Wir vermuten es, denn die Weichen sind richtig gestellt. Die Ganzheitsund Komplementärmedizin ist in
Bern gut vertreten. Neben dem Gesundheitsminister gibt es viele Parlamentarier, die den Volkswillen respektieren und den Verfassungsauftrag korrekt umsetzen wollen, den der Souverän in der eidgenössischen Abstimmung formuliert hat. In der Praxis bedeutet das, dass die Komplementärmedizin neben der Schulmedizin eine wichtige Rolle in der Gesundheitsversorgung der Schweiz in der Zukunft spielen wird. Im Zentrum wird nach wie vor die Schulmedizin stehen, aber es braucht das Miteinander der beiden Therapierichtungen. Deshalb ist auch die Integration der Naturheilkunde in die Ausbildung der Ärzte wichtig. Die ist im Medizinalberufegesetz so geplant.

Man hat uns vorgeworfen, dass wir eine Vormachtstellung der Komplementärmedizin wollen. Das ist aber falsch. Wir wollen, dass sie in der Ausbildung der jungen Mediziner einen Platz hat. Wenn man gemeinsam zusammenarbeitet - schul- und komplementärmedizinisch -, ist das im Interesse der Patienten. Auch müssen alle Ärzte Basiskenntnisse der Komplementärmedizin haben, damit sie ein fachliches Urteil über Methoden fällen könnnen, die bei den Patienten beliebt sind.

Was die Ausbildung der Ärzte betrifft, liegt der Ball recht schnell bei den Universitäten und dann zieht sich das Verfahren in die Länge?

\section{KARGER}


Das ist ein ungelöstes Thema. Es heisst zwar, dass die Vorgaben des Gesetzes übernommen werden müssen, aber was die zuständigen Stellen der Universitäten effektiv umsetzen werden, ist offen. Es ist tatsächlich so, dass die Kompetenz nachher auf Stufe einer Kommission liegt. Je nachdem, wie die zusammengesetzt ist, kann das dann etwas länger dauern, bis die gesetzlichen Erfordernisse umgesetzt werden. Wir werden ihnen auf die Finger schauen und gegebenenfalls die Parlamentsmitglieder über Fehlentwicklungen informieren.

\section{Wie lange wird es voraussichtlich dauern, bis die Komplementär- medizin in der ärztlichen Praxis so verankert ist, wie es der Verfassungsartikel verlangt?}

Der Prozess der Schaffung des Gesetzes und bis die Verordnungen nachher erlassen und in Kraft gesetzt sind, dauert 4-5 Jahre. Anschliessend müssen noch die Leute ausgebildet werden. Es wird 10 Jahre dauern, bis da eine neue Generation herangewachsen ist, die die notwendigen

Walter Stïdeli (46) ist seit 2004 selbstständiger Politik- und Kampagnenberater im Gesundheitswesen. Seine Firma Köhler, Stüdeli \& Partner GmbH (www.koest.ch) betreut unter anderem die Geschäftsstellen des Dachverbandes Komplementärmedizin (Dakomed; www.dakomed.ch) und des Schweizerischen Verbandes für Komplementärmedizinische Heilmittel (SVKH; www.svkh.ch). Aufgabe des Dakomed ist die Umsetzung der Kernforderungen, die mit dem Verfassungsartikel 118a Komplementärmedizin verbunden sind. Der SVKH wiederum setzt sich für marktund wettbewerbskonforme Rahmenbedingungen für Hersteller und Distributoren komplementärmedizinischer und phytotherapeutischer Produkte in der Schweiz ein. Ziel ist es, die Arzneimittelvielfalt in der Schweiz im Interesse der Patientinnen und Patienten zu erhalten. Eine wichtige Aufgabe im Jahr 2013 ist die Unterstützung des Parlaments mit sachspezifischer Expertise bei der zweiten ordentlichen Revision des Heilmittelgesetzes.
Kenntnisse besitzt, um die Komplementärmedizin neben der Schulmedizin kompetent zu beurteilen. Aber schon heute ist die jüngere Generation der Ärzte, die gerade ihre Ausbildung macht, viel offener für Anliegen, die den Patienten beschäftigen. Dazu gehört auch die Komplementärmedizin.

\section{Heute ist also bereits ein Wandel im Gange, der die Komplementär- medizin stärker gewichtet?}

Ja, im ärztlichen Bereich sind zwei Entwicklungen festzustellen, die Zeichen für einen sehr starken Wandel sind. Die eine ist natürlich, dass die Zahl der Hausärzte abnimmt - und damit auch die Zahl der komplementärmedizinisch tätigen Ärzte. Dies ist einerseits unsere Sorge, andererseits besteht Hoffnung, dass es immer genügend qualifizierte Therapeuten gibt. Dort haben wir in der Schweiz einen Wachstumsbereich. Weil es immer mehr chronisch kranke Menschen mit Mehrfacherkrankungen gibt, braucht es mehr Kooperationen. Deshalb wird sich auch die Frage nach der vermehrten Zusammenarbeit von Ärzten und Therapeuten öfters stellen. Und es besteht auch die Hoffnung, dass die Hausarztmedizin insgesamt wieder attraktiver wird, wenn auch der komplementärmedizinische Hausarzt ein attraktiveres Berufsfeld wird. Aber derzeit ist eine Verlagerung in den nichtärztlichen Bereich festzustellen.

\section{Ist das problematisch aus Ihrer Sicht?}

Ja und Nein. Es braucht immer Ärzte, die komplementärmedizinisch tätig sind. Nur so kann die Komplementärmedizin auch Teil der Grundversicherung sein. Deshalb ist es wichtig, dass sie an den Universitäten während des Medizinstudiums gelehrt werden muss. Sie muss zum Bestandteil der «etablierten» Medizin werden. Ob fünf komplementärmedizinische Me- thoden in der Grundversicherung sind oder ob diese fünf von $150 \mathrm{Me}$ thoden in der Zusatzversicherung sind, ist in der Beurteilung und der Wahrnehmung der Öffentlichkeit ein Unterschied.

Besteht die Gefahr, dass die Komplementärmedizin zu einer Art Medizin für die Bessergestellten wird, wenn sie im nichtärztlichen Bereich stattfindet und durch Zusatzversicherungen bezahlt wird?

Eher ja. Die Komplementärmedizin muss in der Grundversicherung sein, damit alle sie nutzen können, die sie benötigen. Sie ist ein wichtiger Teil des Solidaritätsgedankens der Grundversicherung. Zusatzversicherungen können sich nicht alle Menschen leisten und kranke und alte Personen können keine abschliessen.

\section{Worauf ist es zurückzuführen, dass die Komplementärmedizin in Bern heute so viele Fürsprecher hat?}

Im Bundesrat war bereits der ehemalige Gesundheitsminister Burkhalter unseren Anliegen gegenüber sehr positiv eingestellt. Und der heutige Bundesrat Alain Berset war schon vor seiner Wahl sehr engagiert auf diesem Gebiet und hat sich auch am Abstimmungskampf um den Verfassungsartikel beteiligt. Er war Mitglied im Kantonal-Komitee Freiburg, noch bevor er in die Regierung gewählt wurde. Schon damals gab es Kontakte mit dem heutigen Gesundheitsminister, wobei natürlich klar ist, dass ein linker Gesundheitsminister den Anliegen der Komplementärmedizin generell offener gegenüber eingestellt ist als ein Bürgerlicher, aber auch bei den Bürgerlichen gibt es viele offene Personen.

Auch im Parlament hat sich einiges getan. Wir können feststellen, dass in der aktuellen Legislatur, die vor einem Jahr angelaufen ist, die Zahl der Mit- 
glieder der parlamentarischen Gruppe «Komplementärmedizin» zugenommen hat. Die Abstützung ist breiter geworden. Auch bei den Bürgerlichen finden wir Unterstützung, die wir unbedingt benötigen, um Mehrheiten zu finden.

Wenn man sich die Nachwuchssituation anschaut, wie schätzen Sie das Interesse von Schulmedizinstudenten ein, Zeit und Energie in das Thema Komplementär- und Ganzheitsmedizin zu investieren?

Ich denke, dass die jungen Mediziner offener für komplementärmedizinische Angebote sind, weil sie den Patienten ernster nehmen, und es ist völlig klar, dass die Patienten in der Schweiz die Komplementärmedizin wollen. Der Verfassungsartikel wurde mit 67\%iger Zustimmung angenommen, und es wird eine stärkere Ausrichtung an den Bedürfnissen der Patienten geben. Das ist auch bedingt durch den Wechsel von einem paternalistischen System, wo der Arzt weiss, was gut für seinen Patienten ist, zu einem partnerschaftlichen und dialogorientierten System. Also: Es wird zwingend ein zunehmendes Interesse bei den Hausärzten, bei den Schulmedizinern geben, wahrscheinlich weniger bei den Fachärzten. Aber auch dort gibt es Gebiete, wo das Interesse am Steigen ist, wie etwa in der Onkologie. Dort interessiert man sich sehr stark für die Komplementärmedizin.

Jetzt wollen ja gerade junge Leute nicht unbedingt Hausarztpraxen übernehmen, sondern gehen mehr in den Spitalbereich hinein, wo sie geregeltere Arbeitszeiten haben.

Das heutige Hausarztmodell, dass ein Arzt eine Praxis führt oder vielleicht zwei zusammen eine Praxis führen, ist ein Auslaufmodell. Der Hausarzt von morgen wird eine Frau sein. Es werden mehr Frauen die Hausarzt-

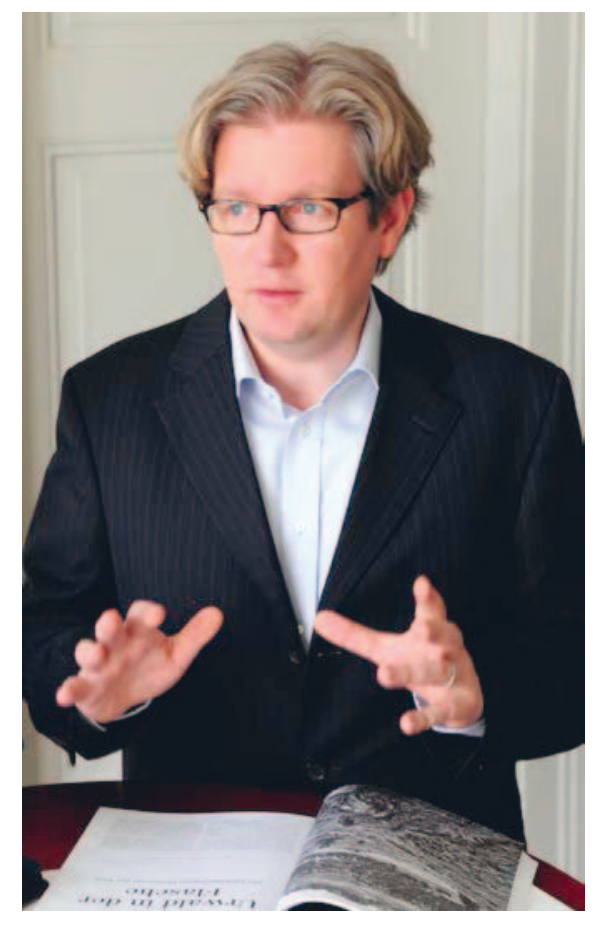

wir haben immer mehr Krankheiten gleichzeitig, und es sind eben mehr Personen am Behandlungsprozess beteiligt - und insofern braucht es einfach einen Gatekeeper oder Koordinator. Und ob das zwingend ein Arzt sein muss oder auch ein Pflegender oder ein nichtärztlicher Therapeut, das wird die Zukunft weisen.

Ein Gesundheitsmanager, der für den jeweiligen Fall die richtigen Leute hinzuzieht?

Genau. Der Begriff «Gesundheitsmanager» gefällt mir persönlich gut. Heute haben die Ärzte einen Anreiz, ihre Leistungen zu verkaufen. Sie verdienen nur durch das Verkaufen von Leistungen, und je mehr sie verkaufen, desto mehr verdienen sie. Und es müsste auch eine Art Paradigmenwechsel geben, den die ManagedCare-Vorlage mit sich gebracht hätte. Ärzte und andere Behandelnde müssen ein ärztliches und ein finanzielles Interesse haben, dass die Patienten rasch gesund werden oder gesund bleiben. so regeln kann, dass die Hausärzte mit den Tarifen zufrieden sind.

Sie erwarten, dass es Hausarztzentren gibt, in denen eine Reihe von Ärztinnen angestellt ist, die dann Komplementärmedizin als Spezialität anbieten?

Das ist ein mögliches Modell. Ob diese integrierte Versorgung physisch an einem Ort in einem Zentrum stattfindet oder ob das dezentral geregelt ist und man an verschiedenen Orten zusammenarbeitet, ist offen. Das Modell funktioniert sicher in den Städten; auf dem Land ist das etwas schwieriger. Ich denke, es geht in die Richtung eines Gatekeepers, der seine Patienten kennt und der dann die ganze Behandlung steuert.

Es ist nicht nur so, dass sich die Versorgung oder die Hausärzte ändern, sondern auch die Struktur der Patienten. Wir werden immer älter,
Also das alte Modell der Antike, wo Ärzte für die Gesundheit ihrer Patienten bezahlt wurden und nicht für die Behandlung von Krankheiten?

Falls es das wirklich gab und nicht exklusiv dem Kaiser vorbehalten war, wäre das wohl ein gutes Modell.

Die Veränderung der Patientenstruktur bedingt ja auch Veränderungen im Anforderungsprofil der Ärzte. Das heisst, sie müssen offener sein; das, was Sie als nichtpaternalistisches System angesprochen haben. Wie erleben Sie eigentlich Ärzte beim Umgang mit diesen Veränderungen?

Ich denke, es ist schon schwierig für einen Arzt, sich nach langer schulmedizinischer Ausbildung an der 
Universität und nach langer Assistenzzeit einzugestehen, dass das, was er gelernt und praktiziert hat, Grenzen hat und dass es keine Allheilmittel gibt. Es gibt Ärzte, die offen sind und nach Alternativen Ausschau halten. Viele von ihnen beschäftigen sich dann mit der Komplementärmedizin.

Der Durchschnitt der Hausärzte ist heute zwischen 55 und 60 Jahre alt. Sie werden ihr Verhalten nicht mehr grundlegend ändern, was in Ordnung ist. Die jungen Hausärzte werden offener sein.

Die Nachfrage an Komplementärmedizin und ganzheitlicher Betrachtung des Lebens wird wachsen, auch weil die Bevölkerung heute viel gesundheitsbewusster ist?

Ja, ganz klar. Wer sich um seine eigene Gesundheit kümmert, wer sich für gesundes Essen interessiert, für Bewegung, für Sport, für Gesundheit, der ist effektiv auch gesünder, der hält sich auch eher an die Vorgaben oder eben an die Abmachungen und die Empfehlungen des Arztes oder des Therapeuten. Solche Menschen wissen, dass der eigene Anteil an der Gesundheit ein ganz wichtiger ist. Die Rolle der Behandelnden besteht auch darin, die Befähigung der Patienten zu stärken, damit Krankheiten möglichst nicht eintreten.

Welche Rolle spielt die europäische Gesetzgebung für die Schweizer Komplementärmedizin und können Ärzte in der Schweiz davon profitieren?

Im ärztlichen Bereich ist die Rolle der Gesetzgebung in Europa sehr bescheiden. Wenn es um die Heilmittel geht, ist die Bedeutung hingegen sehr gross. Gerade bei den Produkten, die arzneimittelähnlich sind, wie z.B. Medizinprodukte, Lebensmittel und Nahrungsergänzungsmittel, übernehmen wir die europäischen Regelungen eins zu eins. Diese sind dann zum
Teil im Vollzug noch strenger als jene in einzelnen EU-Ländern. Der Einfluss auf zugelassene Arzneimittel ist enorm: Bei der Komplementärmedizin kämpfen wir dafür, dass es auch spezifische und vereinfachte Zulassungsmöglichkeiten gibt. Schliesslich haben wir die Komplementärmedizin in der Verfassung explizit verankert.

Wie sieht die wirtschaftliche Situation der Hersteller von komplementärmedizinischen Medikamenten oder Wirkstoffen aus?

Das ist unterschiedlich zu beurteilen. Wer als Hersteller Produkte aus der Klassischen Homöopathie produziert, hat heute Schwierigkeiten. Aufgrund der behördlichen Restriktionen kann er nicht mehr ein Vollsortiment anbieten. Deshalb sind viele Produkte vom Schweizer Markt verschwunden. Dabei werden die Produkte nach wie vor verlangt. Ärzte können sie legal einführen. Nichtärztliche Therapeuten importieren sie oft auf verschlungenen Wegen. Es ist ein riesiger Grauund Schwarzmarkt entstanden, und wenn man halt nicht mehr die ganzen Produktlinien in der Schweiz kaufen kann, weicht man auf ausländische Hersteller aus. Bei denen ist die Qualität zum Teil nicht sichergestellt, weil diese nicht durch Swissmedic geprüft wird.

\section{Warum können die Schweizer Hersteller nicht mehr ihr ganzes Sortiment anbieten?}

Die Zulassungskriterien sind teilweise viel zu hoch. Wenn Sie ein Produkt haben, das Sie zehnmal im Jahr verkaufen können, können sie nicht 1000 Franken für eine Zulassung bezahlen und weitere Belege und Nachweise liefern. Das Verfahren wird einfach zu aufwendig und die Gesamtkosten sind im Vergleich zum Umsatz zu hoch. Was wir dem Gesetzgeber vorschlagen und was auch der Bun- desrat in seine Botschaft übernommen hat, ist die sogenannte Kleinmengenlösung: 100 Einheiten pro Jahr dürften ohne Zulassung verkauft werden. Die Chancen sind meiner Meinung nach gross, dass dieser Vorschlag eine Mehrheit findet.

Welche sind die wichtigsten Themen der Komplementärmedizin für die Branche in den nächsten 1-2 Jahren?

Wirklich zentral im 2013 ist die Frage des Heilmittelgesetzes. Und dort ist eine Stärkung der Komplementärmedizin wichtig, indem die Zulassungshürden weiter heruntergeschraubt werden. Was wir uns auch vorstellen können, ist, dass Produkte, die eine Swissmedic-Zulassung haben, mit einem Qualitäts-Label besonders gekennzeichnet werden. Patienten sind heute oft überfordert. Sie kennen die Unterschiede zwischen Arzneimitteln, Medizinprodukten und Nahrungsergänzungsmitteln nicht. Dort muss die Komplementärmedizin gestärkt und die Zulassung vereinfacht werden.

Es ist ja eine sehr paradoxe Entwicklung, dass insgesamt das Interesse an der Komplementärmedizin enorm zunimmt, die Nachfrage steigt und andererseits aber auf der Wirkstoffebene und beim Angebot an Produkten eine Erosion stattfindet.

Eine grosse Gefahr ist auch, dass irgendwann die Gegner recht bekommen, die sagen, das wirkt ja nicht, wenn gar keine oder fast keine Wirkstoffe oder keine aktiven Stoffe mehr drin sind. Das ist ein Problem, und wir hoffen wirklich, dass wir jetzt durch die zweite ordentliche Revision des Arzneimittelgesetzes dort gegensteuern können und dass das Parlament unsere Forderungen umsetzt, die namentlich auch in einer parlamentarischen Initiative formuliert wurden. 